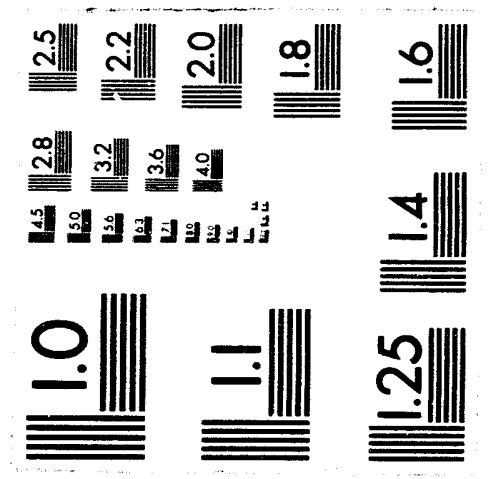



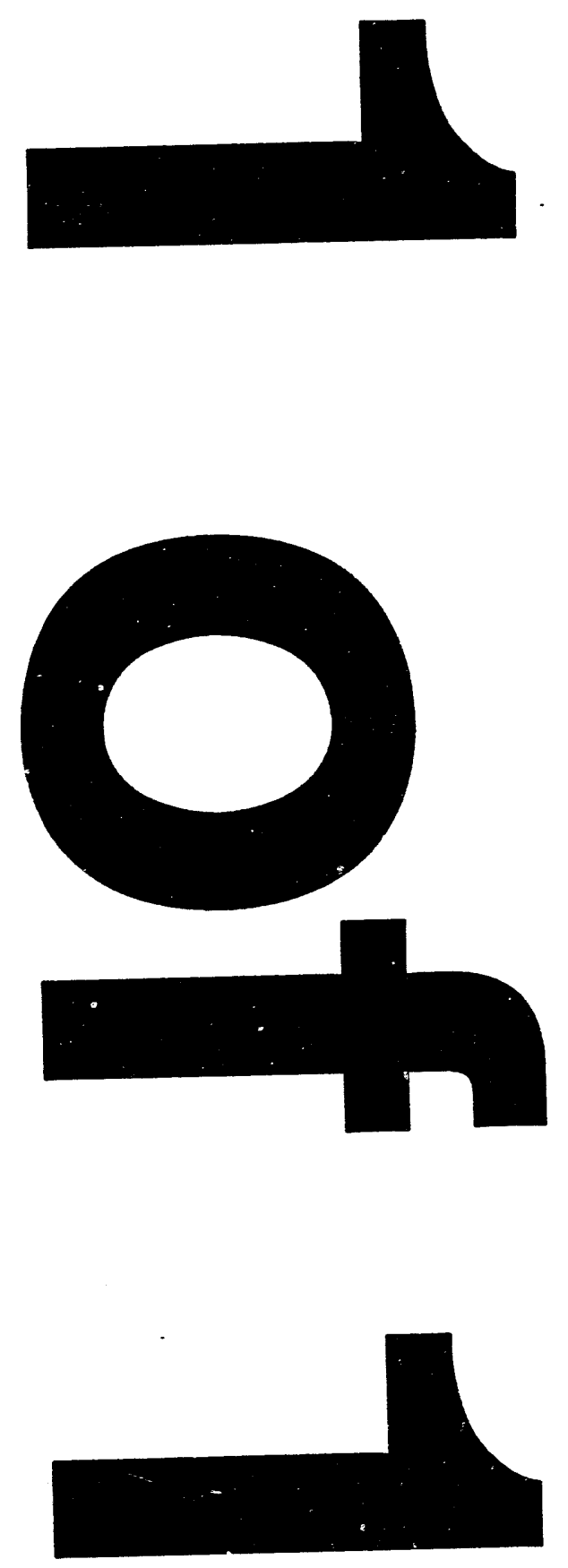


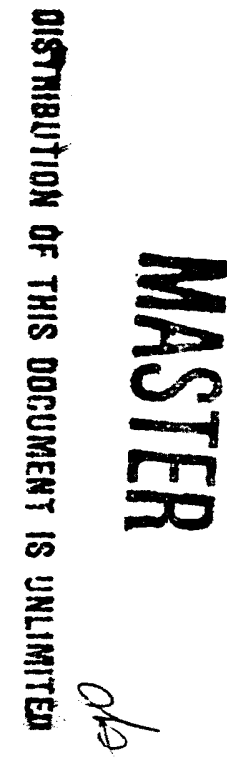

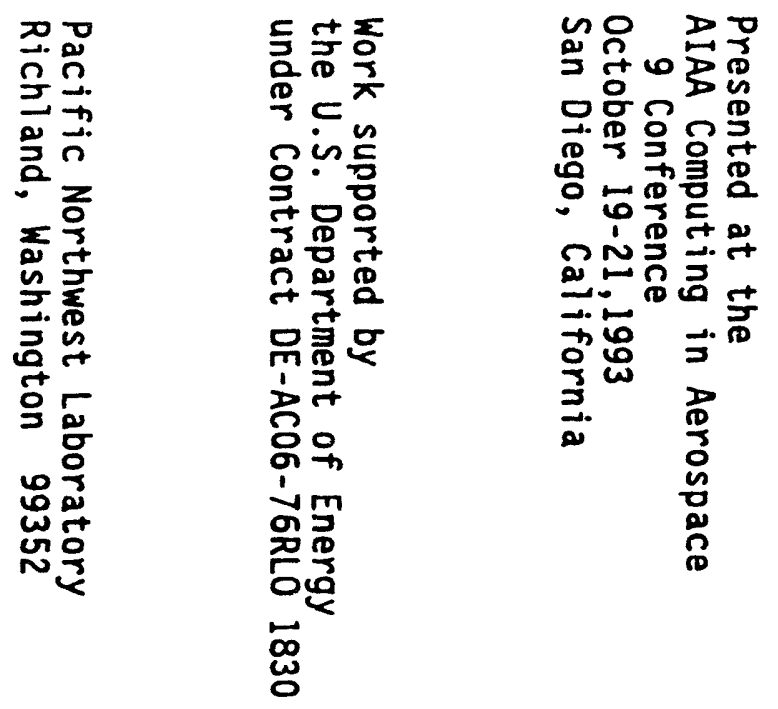

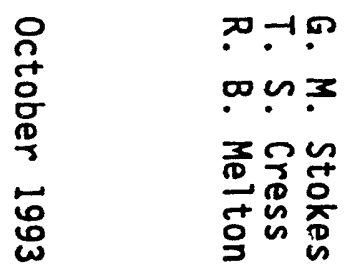

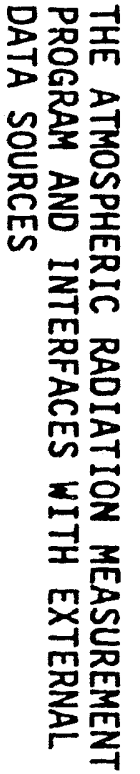

\section{DISCLAIMER}

This This report was Government. Neither the United States Goves implied, or assumes any legal liability or responsiemployees, makes any warranty, express or impled, of any information, apparatus, product, or bility for the accuracy, completeness, or usefulness of any inform privaty owned rights. Referprese that its use would not infringe privately owned rese Re process disclosed, or secific commercial product, process, or service by trade neme, trademark ence herein to any specific constitute or imply its endorsement, recommanufacturer, or otherwise does not States Government or any agency thereof. The views mendation, or favoring by the United Stales Govectessarily state or reflect those of the and opinions of authors expressed herein do not

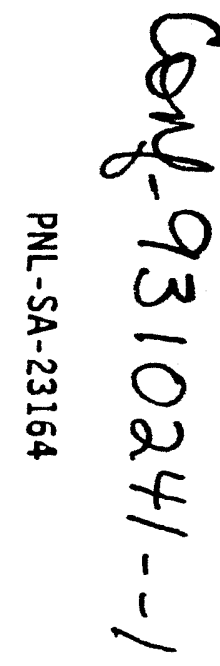




\title{
- ATMOSPHERIC RADIATION MEASUREMENT PROGRAM AND INTERFACES WITH EXTERNAL DATA SOURCES
}

\author{
G.M. Stokes, T.S. Cress, and R. B. Melton \\ Pacific Northwest Laboratorya \\ Richland, Washington, U.S.A.
}

\section{INTRODUCTION}

The Atmospheric Radiation Measurement (ARM) Program is the Department of Energy's major field project in support of its global change research program. Its objective is to improve the performance of cloud and radiation models and parameterizations in general circulation models (GCMs) used for climate research. The data produced by ARM will be handled and maintained to satisfy the research needs of the program and to be accessible and usable by the general research and academic communities. In addition to data from field instrumentation, ARM Science Team needs include a substantial amount of data from outside the ARM Program ("external data"), which will be acquired and provided routinely through the ARM data system. With respect to data archival and sharing, the ARM Program reflects the objectives delineated by the U.S. Global Change

Research Program (USGCRP) and reflected in the design of the Global Change Data and Information System (GCDIS). The purpose of this presentation is to summarize the conceptual designs embodied in the ARM data system and the status of its implementation.

The ARM Program's focus on cloud and radiative processes and their associated feedbacks, like many global change research issues, embraces a set of objectives that require multidisciplinary approaches. Data streams from state-of-the-art ground-based and airborne in situ and remote sensors are clearly required, some with very high data rates. For some data needs, new instrument capabilities are required and may involve either exploiting current technologies or developing new observational concepts. External data requirements include satellite soundings and imagery, output from research and operational models, and routine atmospheric observational data from the National Weather Service. Observational data from demonstration instrumentation of several National Oceanic and Atmospheric

Administration (NOAA) laboratories, while still developmental are, in some cases, the state-ofthe-art data streams required to satisfy research objectives of the Science Team.

The ARM Program is feasible because the development of dara processing capabilities and instruments has advanced to where large volumes of high resolution data can be routinely acquired, quality controlled, processed and moved to distant users by high speed communications capabilities. Unlike the very recent past, telecommunications technologies now permit the scientist to access a broad range of data from his desk. It was within these developing realities that the concepts for the ARM Program were developed and are being implemented. These concepis are very much a part of the philosophy of the USGCRP.

\section{ISSUES IN DATA UARINC AND ACCESSLILITY}

Large rese arc' pryans a y broad ranges of scien of acists sch as many

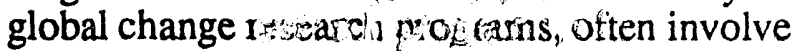
multidisciplinary porristres and require routine access a $v$. seriety of data streams. Some data stuans dy electronically available in resil tin, wilite others require access through $\mathrm{w}$ : or processing centers, or throng u Lications relays. Some data have siripleserture and are easily handled, but others have complex, multi-

aPacific Northwest Laboratory is operated for the U.S. Department of Energy by Battelle Memorial Institute under Contract DE-AC06-76RLO 1830. 
dimensional structure and require specific formatting and processing. Hardware, telecommunications and data formatting practices and standards significantly impact the accessibility of data electronically and the level of effort required to use it.

The evolving expectation is that data will be available or can be made available regardless of its source. The USGCRP philosophy is that global change research data will be available, with some minor caveats. A complicating factor, however, is that broadly based research efforts under the USGCRP will need access to data and research results from non-USGCRP programs. Access to these data requires special effort, the investment of time and resources, and a lot of cooperation. Unlike the recent past, data production or archival activities, whether part of the USGCRP or not, now need to recognize that data sharing and transfer will become routine, and that large-scale research programs often can use data as fast as it is produced. Projects, such as ARM, are increasingly including design considerations that will permit routine, straightforward access to highly diverse and large data sets through well-documented and prescribed procedures. Considerations that art increasingly common include timely access, quality controlling data in real time, documentation of data and data formats, and accessibility of documentation.

The ARM data environment was conceived to adhere to the general principle of the USGCRP for early data availability and the provision of data upon request to the general scientific community. Beyond the general principles of the USGCRP, the more specific implementation recommendations for the GCDIS embody many of these same needs and considerations. As a result, the ARM Program is well positioned to implement the principles of the GCDIS; many already are being implemented.

\section{THE ARM DESIGN}

The fundamental concept of the data system design for the ARM Program is to provide quality data to each member of the ARM Science Team in a form and on a time scale determined by the scientist, and to a location of his or her designation. Beyond the needs of the Science Team, and in accordance with the principles of data management for the USGCRP, ARM data will be archived for access by the general scientific community.

The fundamental data needs of the ARM Program are determined from what is required to support the research objectives of the Science Team. These requirements are determined by preparing for each Science Team member an "Experiment Design," a document that identifies the data needs of the scientist as well as where, when and in what format data are to be delivered. The data required will originate from project instrumentation, from data processing algorithms being run routinely within the ARM data system, or from external data, such as satellite imagery, acquired largely through automated "hands off" interfaces with other electronic databases.

ARM has designed a data processing and transfer capability that meets the needs of the Science Team, and supports the larger USGCRP research community. In achieving these objectives, the design and implementation of the system followed a number of guiding principles that helped shape some aspects of the current configuration. These include

- Arm will evelop as little as possible; instead existing capabilities will be used, such as off-the-shelf instrumentation or software, and adapted to the needs of ARM.

- $\quad$ ARM data will be accessible by all ARM participants equally, and, through the Archive, by the general scientific community.

- ARM sites will acquire data routinely for transmission to Science Team members in a manner most appropriate to each individual scientist. Scientists should feel that the data received are of the same quality as if they were there to take it.

- The data system will acquire all routinely re quired external data and provide that data to appropriate participants; external data will be accepted with the quality control exercised by the originator. 
- The quality of ARM data will be assessed and documented such that the user can understand the data and the instrumentation that provided it.

- All capabilities will be implemented to permit the earliest possible use for data acquisition and provision to the Science Team: implementation will be phased to permit using components of the instrumentation and data systems that are available at any given point.
- All project-originated data will be archived in raw and processed form to the greatest extent possible. External data archived elsewhere will not be maintained except where reacquisition would be difficult, such as some satellite data extractions for site areas.

The fundamental operational component of the ARM Program is the Cloud and Radiation Testbed (CART); i.e. the instruments, data system and other facilities necessary to acquire the data, process it, deliver it, and store it.

Figure 1 shows the basic schematic structure of the system being implemented.

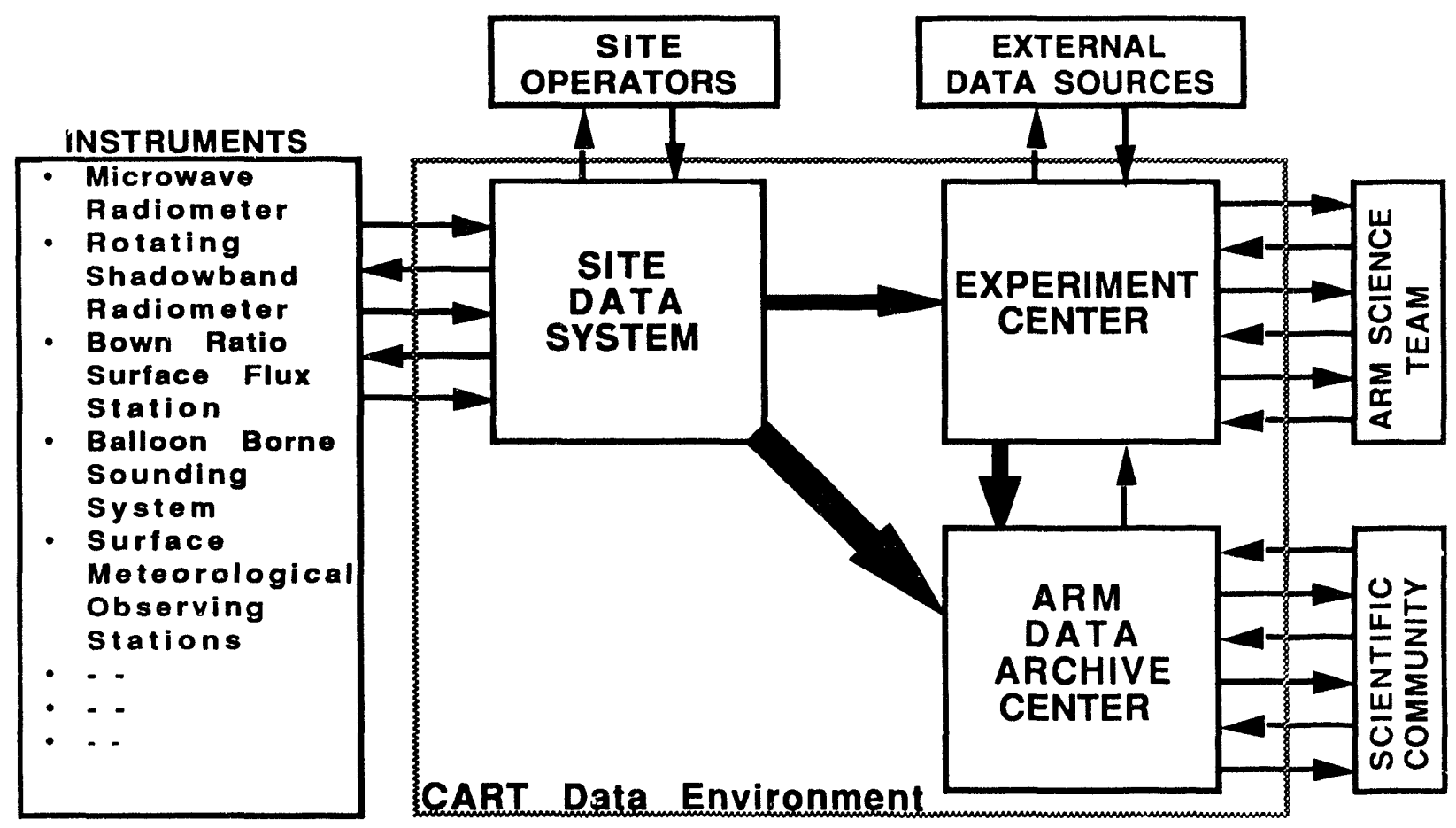

FIGURE 1. Schematic representation of ARM data system components with associated data flows.

\section{SITE DATA SYSTEM}

The Site Data System (SDS) acquires data from site instruments, conducts the first levels of quality control as the data are acquired, and transmits data files to the Experiment Center as well as directly to the Archive. The SDS is based on the Zeb data integration system developed by National Center for Atmospheric Research (NCAR) for use in field programs where data from a variety of disparate sources needs to be captured, processed and displayed. Appropriate to campaign-style efforts of limited extent and duration, Zeb has required further development to be an effective operational system, capable of handling the larger and more continuous data streams of ARM. ARM is working with NCAR to extend and adapt Zeb's capabilities. At the site level, data quality assessments are performed automatically to ensure that the data from an instrument is what should be expected in the absence of other, directly comparable data. This includes basic 
limit checks, consistency, and basic pattern behavior, such as a trend due to a diurnal cycle. A basic function of the SDS is to ensure that the individual instruments are performing as expected and that the data transmitted from the site are the most accurate that can be routinely generated by individual instruments. Calibration and other instrument data of importance are retained in the data system for inclusion as "metadata" (data about the data) with the data and is archived as such. Questionable data is retained in the data stream, but appropriately flagged and documented. Ancillary information that may help understand or interpret instrument data, such as human observations of sky and surface conditions around instrument sites, are routinely entered in electronic logs and represent a further source of data and metadata data.

\section{EXPERIMENT CENTER}

The Experiment Center is the crossroads for data acquisition (inflow) and delivery (outflow) to the Science Team and the Archive. The Experiment Center acquires data from each of the active sites, performs quality assessment procedures, acquires external data, executes algorithms to produce derived data streams from observational data, packages and transmits data to Science Team members, and transmits all quality assessed data and derived data to the Archive. After receiving data from a site, the Experiment Center is intended to perform a second level of automated quality assessment involving intercomparisons of data from several complementary instruments or possibly to model generated data, e.g., radiometric observations compared to radiometric model predictions. This level of assessment is focused on ensuring that the integrated data set from a site is self consistent and that the data streams from individual instruments are accurately representing the state of the atmosphere and its radiometric conditions. This activity may include the performance of Quality Measurement Experiments to more fully investigate the accuracy or representativeness of given data streams by more in-depth comparisons than are part of the automated quality assessment procedures. In producing data for transmission to the Science Team, standard formats are encouraged, but not required. The Network
Common Data Format (NetCDF), for example, is capable of accommodating vector data as well as metadata. NetCDF is the most common format used for transmission of ARM data to users. Satellite imagery is typically acquired in HDF format, and this is retained in packaging data for Science Team members. The intent of ARM, however, is to provide data to the user in the most usable form for that user. In some cases, this means that ASCII is the preferred format, and the data will be provided in that format if at all possible.

\section{ARCHIVE}

The Archive receives data files and activity logs from the sites and the Experiment Center, as well as instrument data that is recorded in other documents in the data system. These data include instrument maintenance and calibration data, subjective observational data captured in logs, data derived from external sources, and information describing quality checks and algorithms, for instance. In addition, the Archive captures and retains other project records and documents, whether or not in electronic form. The role of the Archive encompasses several types of activity. The Archive maintains and safeguards all raw and processed data generated by the ARM instruments and algorithms, as well as all associated "information" about the data (e.g. metadata cata) that may be necessary to understand or interpret the data. Secondly, the Archive is to function as the "window" for ARM to the outside world, and the general scientific community beyond ARM participants; this will include academic interactions and interactions with other data centers.

At this time, the Archive is not fully implemented. Currently, all data are being captured and held in temiporary media. The Archive is capable of responding to data requests, but the response is manual and delay can be expected. Hardware is being put in place for the sizable task of handling the large volume of data to be retained, up to 100 terabytes of data and metadata, and for providing access to the Archive. The Archive will eventually use a tape-based mass storage system based on the National Storage Laboratory architecture. For initial impiementation, the user interface will be 
designed to support professional researchers in the atmospheric sciences and related disciplines. The ultimate design and mode of operation will benefit the larger community with a user interface designed to provide enough information about data and products in the ARM Archive so that users can explicitly identify what they need. Science Team members access Archived data through the Experiment Design process in response to which the Experiment Center will access the archived data, process it, and provide the required results to the Science Team member in the same manner as if it were current data.

\section{DATA SHARING EXPECTATIONS}

The USGCRP policy for data management includes the principles of early and continuing accessibility of high-quality, long- term data sets, and the full and open sharing of data for global change research. For ARM, the implementation of these principles has two aspects: the availability of ARM data to the general scientific community, and the availability of data and research results from other programs for use by members of the ARM Science Team.

Access to ARM data is required by a variety of users ranging from those within the program through collaborating programs to those independent of ARM. Within ARM, the operational infrastructure concerned with data acquisition and provision involves eight $\mathrm{DOE}$ laboratories, four laboratories of other Federal agencies, and at least two universities.

Activities in the infrastructure typically involve monitoring or working with specific sources of data, such as specific instruments, or developing and implementing new data processing procedures. In general, data requirements to support these functions are met by direct access to the SDS or routine direct delivery of data from the SDS to the functional user. Science Team needs for data are met through the normal functions of the Experiment Center in accordance with the Experiment Design established for each Science Team participant. Collaborating program needs, such as in a campaign, or for the evaluation of a "guest" instrument at one of the sites, will be met on a case-by-case basis. In general, once a collaboration is established, an Experiment
Design will be established in the same manner as for a Science Team member. The Experiment Design will specify the data that is required, when it is required, its format, and how it will be delivered. Generally, data will be supplied electronically from the Experiment Center, but circumstances and the needs of the collaborator may make it more desirable, alternatively, to furnish the data from the Archive. While a suite of standard procedures will be the basis for setting up these transfers, flexibility to respond to individual circumstances will be maintained.

Access to ARM data by the general scientific community and other data centers will typically be through the Archive. In the near term, response by the Archive will continue to be manual. As it is more fully implemented, the Archive will grow to more closely approximate a user facility where data catalogs and data views are available for browsing. There will be an on-line data request capability. Some selected data will be available for browsing, but requests will typically be filled by accessing tape storage media. With some delay, data will be moved to pickup files or, perhaps, to more desirable media for data transfer than electronic file transfer for the individual request. Extensive documentation on the contents of the Archive and how it can be used most effectively will be available on line. The goal is to be as responsive and "hands-off" as possible, i.e. automated responses to many requests.

Access to external data sources by ARM is not so straight-forward and embodies a range of experience to date. The sources for data required by $A R M$ investigators include the National Weather Service, the National Environmental Satellite Data and Information Service, other satellite data (such as the defense meteorological satellite microwave data and some imagery) and model results from mesoscale models that are still in development, to identify a few. Typically, these are not data sources within the USGCRP, but our expectations include convenient access if at all possible, cooperation in establishing automated access procedures, minimal cost (if any) and documentation that makes the access useful. Experience is mixed. In general, we have been able to access external data sets through a 
variety of mechanisms, but the path to access has seldom been straightforward or without cost. One of the major shortfalls encountered consistently has been a lack of documentation that optimizes the usability of the data to which we have acquired access. One of the major frustrations experienced is the difficulty of accessing valuable research model results without substantial investments to facilitate such access. We have often experienced a real willingness to establish a more efficient interface capability on the part of data suppliers, but seldom have these suppliers developed their data processing system to facilitate automated data sharing and transfer beyond their immediate user group.
The data management philosophies of the atmospheric sciences community and its data centers are moving in the right direction, but there is substantial progress yet to be made. The weakest ingredient has been adequate implementation of the philosophy being embraced by GCDIS that the agency acquiring data important to USGCRP research activity has a role to make that data available and accessible, and has a responsibility to facilitate the effective use of that data. This is a particular problem for data being acquired and stored outside of the USGCRP. In particular, this is a philosophy that needs to be extended to the national data centers where our experience suggests there is a willingness, but, at least until recently, inadequate support to achieve the objective.

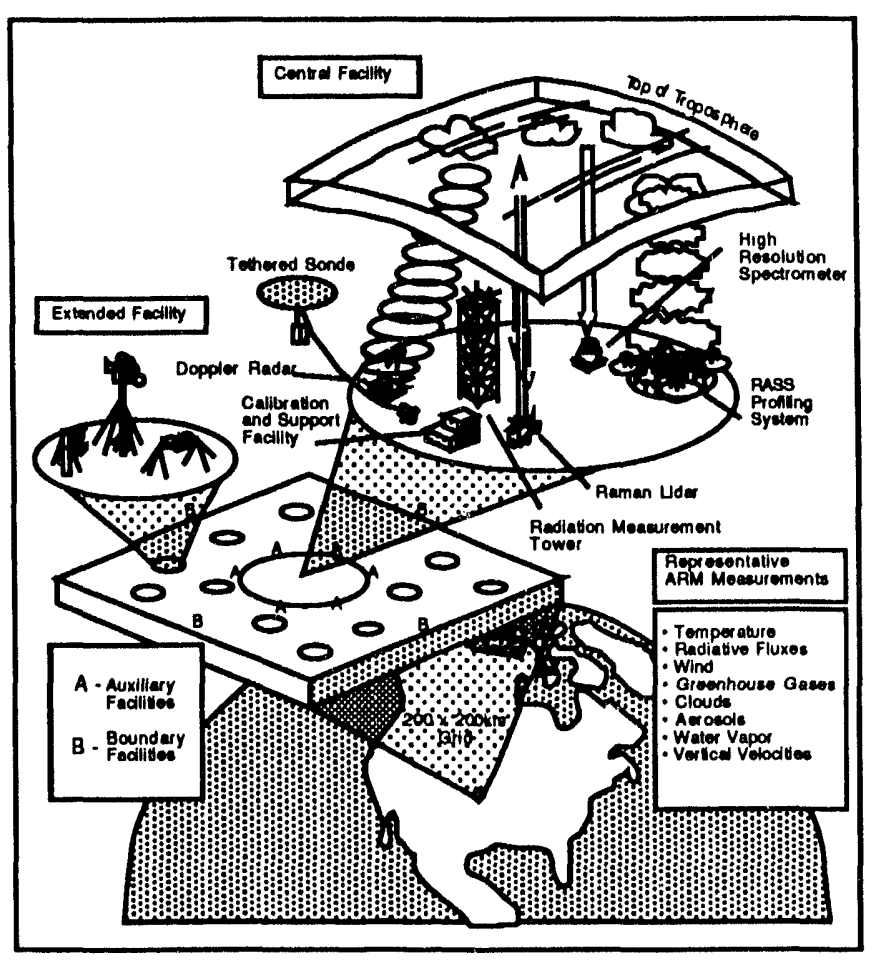

FIGURE 2 Schematic representation of fully implemented CART site.

\section{STATUS OF CART IMPLEMENTATION}

Figure 2 is a schematic representation of a fully implemented CART site. The first CART site is reflective of this design and is now about $80 \%$ operational. The central facility is in place and operational with the latest major instrument additions being installed or tested at this time.
The central facility is focused on the documentation of radiative fluxes and the radiative environment, e.g., presence of clouds, in the atmospheric column above the central facility. The network of extended and boundary facilities intended to document the radiative variability across an area 
representative of a GCM grid cell and to support the development of improved cloud representations will be largely completed by the end of the year. The SDS is fully operational, although data ingest software for the latest instrument systems is still being developed. Some instrument data is being acquired outside of the Zeb data handling system for immediate data availability, but this practice will not be frequent due to the limitations it places on processing and delivering data to a wider set of users. Several Intensive Operational Periods have been implemented based on Central Facility instrumentation and include several unique opportunities to collaborate with other programs that are not directly part of the ARM Program. These include, for instance, efforts by UNAVCO and North Carolina State University to investigate water vapor distribution measurements using GPS signal delays, and by the South Dakota School of Mines to use the ER-2 Advanced Visible and Infrared Imaging Spectrometer (AVIRIS) for surface characterization measurements. Both of these efforts could result in data of high value to the ARM Program and represent at least a segment of the range of programs that we would like to see the CART sites support.

The Experiment Center is, likewise, functioning. Data are being acquired from the Oklahoma site and from a variety of external data sources. In accordance with the operational design, the Experiment Center is providing packaged data to about half of the members of the ARM Science Team. Once routine access to the MAPS model data fields has been established, the number of Science Team members being directly supported will represent most of the Science Team. A number of new Science Team members will be supported as soon as their Experiment Designs can be established and implemented. At this point, many of the automated processes envisioned for the Experiment Center are still being developed and are anticipated for implementation in the next year.

The Archive is capturing all data from the Site and from the Experiment Center. New hardware that will expand the storage and processing capabilities of the Archive substantially; will be put in place over the next year. Until then, the capability of the Archive to respond to data requests will be limited and will be largely manual.

Toward the end of 1994, the modular components being designed to implement measurements at the second CART site in the Tropical Western Pacific will be assembled and tested. Initial deployment will be to one or two island sites. These sites will be largely unmanned and visited only periodically by a maintenance team. The real-time data stream will likely be limited to status data and to a limited sample of the most representative or informative data. Data will be redundantly recorded on site on tape. Tapes will be changed through local arrangements and periodically mailed back to the Experiment Center for processing.

We plan to deploy the third CART site in Alaska about two years following the deployment to the tropical western Pacific Ocean. Preparatory work is already underway with limited instrument tests taking place in the vicinity of Point Barrow.

The last two locales, recommended by our locale recommendation team for long-term measurements have been changed to supplementary locale status. We may conduct limited measurements at those sites sometime in the future, but will not establish long-term CART measurement facilities.

While the ARM Program is addressing the DOE requirement to improve the performance of general circulation models used for climate research, the CART sites represent an opportunity, a research "user facility" in essence, to investigate a much broader range of atmospheric processes than will be addressed within the designed program. The implementation of the CART data system and the broad accessibility of ARM data enhance the CART sites as potential opportunities and represent the essence of the USGCRP philosophy for data sharing and availability. The challenge is to take advantage of that opportunity.

\section{REFERENCES}

U.S. Department of Energy, 1990: Atmospheric Radiation Measurement Program Plan, DOE/ER-0441. 
11
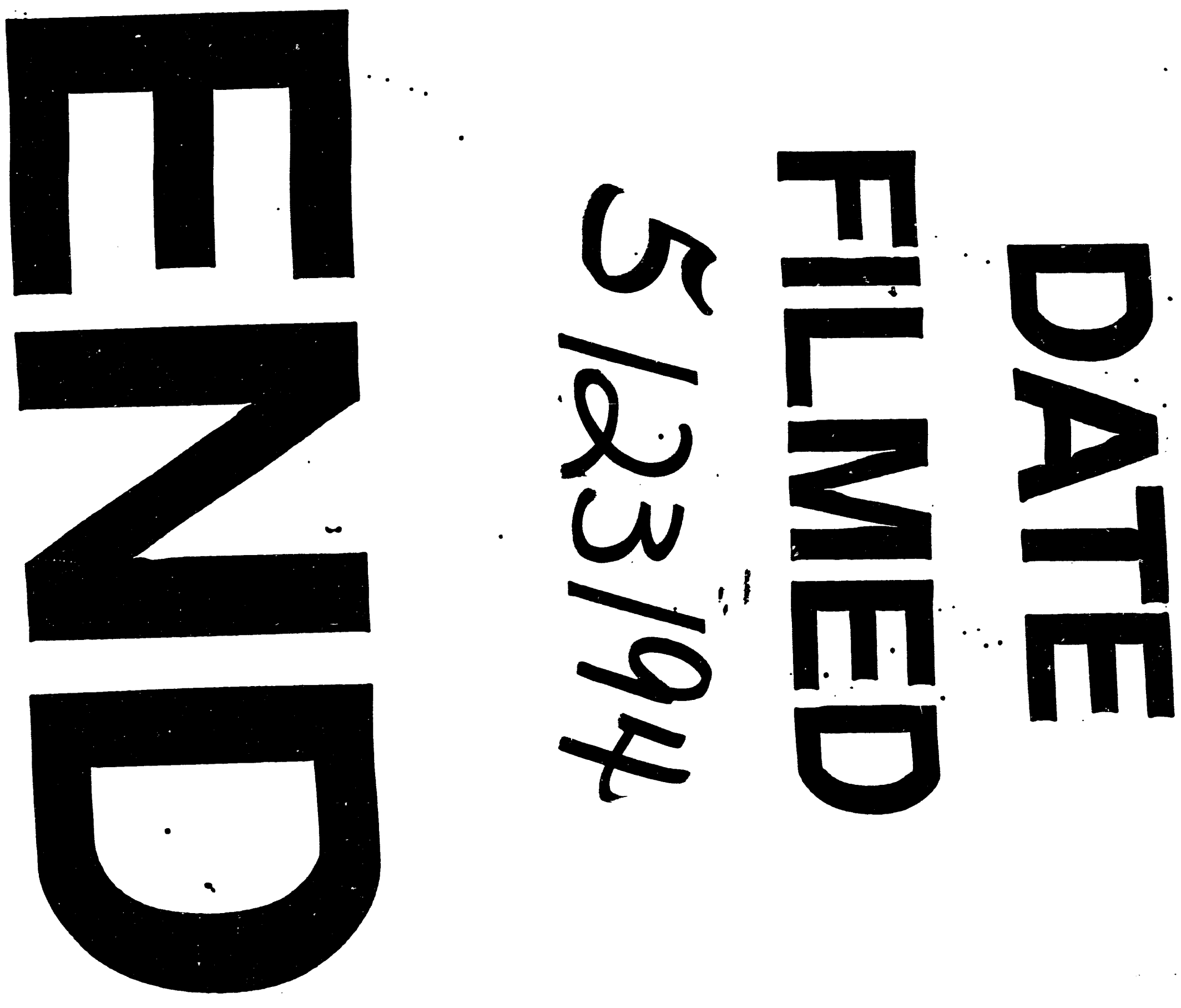


$$
\text { Ham }
$$

1 